Check for updates

Cite this: RSC Adv., 2017, 7, 47704

Received 13th August 2017

Accepted 5th October 2017

DOI: $10.1039 / \mathrm{c} 7 \mathrm{ra0} 0860 \mathrm{k}$

rsc.li/rsc-advances

\section{Multi-functional thermo-crosslinkable collagen- metal nanoparticle composites for tissue regeneration: nanosilver vs. nanogold $\dagger$}

\author{
K. Hosoyama, $\neq^{a}$ M. Ahumada, (D) $\ddagger^{a}$ C. D. McTiernan, ${ }^{a} J$. Bejjani, ${ }^{a}$ F. Variola, ${ }^{b}$ M. Ruel, ${ }^{\text {ac }}$ \\ B. Xu, ${ }^{\text {cd }}$ W. Liang, ${ }^{\text {cd }}$ E. J. Suuronen ${ }^{* a c}$ and E. I. Alarcon (D) *ae \\ Collagen-silver/gold biomimetic matrices were developed for cardiac tissue engineering. The \\ incorporation of nanoparticles led to modifications in the 3D structure of the collagen matrices, with \\ gold increasing the materials' viscosity by $\approx 4$ times. Electrical conductivity was also enhanced upon \\ addition of the nanoparticles. The materials promoted reparative macrophage migration and improved \\ the proliferation and function of neonatal cardiomyocytes under electrical stimulation.
}

Nanomaterials have altered the conventional view of tissue engineering. ${ }^{\mathbf{1 , 2}}$ Over the years, several attempts have been made to prepare biomimetic nano-composites for cardiac tissue engineering. While we have recently reported on the development and use of collagen-derived nanofiber matrices containing embedded silver nanoparticles, ${ }^{3}$ other reports have explored the use of nanotubes ${ }^{4-6}$ and gold..$^{7-10}$ The common goal of such materials is to improve electrical conductivity of the tissue for stimulating functional cardiomyocyte regeneration, ${ }^{\mathbf{1 1}}$ which is lost in the infarcted myocardium due to tissue scarring and cell necrosis. While there has been much progress in the development of these materials, the anti-inflammatory and physical properties imparted to these structures through the incorporation and manipulation of its nano-components remain mostly unknown.

Peptide-modified nanostructures offer numerous advantages over common surface anchoring protecting agents in nanomaterials. For example, peptide stabilized nanomaterials can be chemically tethered to $3 \mathrm{D}$ matrices, where they can be used to impart antimicrobial properties, as we have previously reported for solid corneal implants containing CGS-LL37 modified nanosilver. ${ }^{\mathbf{1 2}}$ However, to this day, the therapeutic effects of using peptide modified nanogold and nanosilver

\footnotetext{
${ }^{a}$ Division of Cardiac Surgery, University of Ottawa Heart Institute, 40 Ruskin Street, Ottawa, Ontario, K1Y 4W7, Canada. E-mail: Esuuronen@ottawaheart.ca; Ealarcon@ottawaheart.ca

${ }^{b}$ Department of Mechanical Engineering, University of Ottawa, Ontario, Canada ${ }^{c}$ Department of Cellular and Molecular Medicine, University of Ottawa, Canada ${ }^{d}$ Cardiac Electrophysiology Lab, University of Ottawa Heart Institute, Canada ${ }^{e}$ Department of Biochemistry, Microbiology, and Immunology, University of Ottawa, Canada

$\dagger$ Electronic supplementary information (ESI) available: Experimental details and supplementary figures. See DOI: $10.1039 / \mathrm{c} 7 \mathrm{ra0} 8960 \mathrm{k}$

\$ Authors contributed equally to this work.
}

within an injectable collagen formulation for cardiac repair remains unexplored. As such, we have prepared and characterized for the first time the electrical and biological properties of a series of hybrid collagen-peptide modified nanosilver and nanogold thermo-crosslinkable materials.

Fig. 1a depicts a schematic representation of the experimental procedure used to prepare the hybrid materials containing the spherical nanostructures (silver or gold, see Fig. S1†). The nanoparticles were mainly spherical in nature with sizes of $11.3 \pm 2.4$ and $7.3 \pm 3.6 \mathrm{~nm}$, for gold and silver, respectively. The nanogold surface was modified using an adapted protocol from our previous work ${ }^{\mathbf{1 3 - 1 6}}$ employing nanosilver (for further experimental details please refer to ESI $\dagger$ ). The CGS-LL37 peptide that has demonstrated higher affinity for nanosilver surfaces leading to the formation of non-toxic, yet bioactive, nanostructures ${ }^{17,18}$ was used to promote nanoparticle coalescence and allowed for the preparation of concentrated stock solutions. Re-suspended nanoparticles retained their characteristic plasmonic absorbance (Fig. S2 $\dagger$ ). Incorporation of the nanomaterials gives rise to the distinctive yellow or red colours of the collagen matrices (Fig. 1a). In addition, the plasmonic absorption bands for both silver $(\approx 420 \mathrm{~nm})$ and gold ( $\approx 540 \mathrm{~nm}$ ) are retained in the collagen matrices (Fig. 1b). These remain stable $(<20 \%$ decrease) after incubation for $24 \mathrm{~h}$ at $37^{\circ} \mathrm{C}$ (Fig. 1b, insets).

Microstructural analysis of the collagen matrices containing the nanoparticles were carried out using low temperature cryoSEM (Fig. 1c). Incorporation of nanosilver within the collagen structure produces noticeable changes in the supramolecular organization of the collagen hydrogel. Of note, at concentrations of nanosilver $>0.025 \mu \mathrm{M}$, a consistent fibril-like structure was observed, whereas upon adding nanoparticulated gold, only a slight increase in the number of fibre-like structures was observed at $0.1 \mu \mathrm{M}$. However, neither silver nor gold 
a

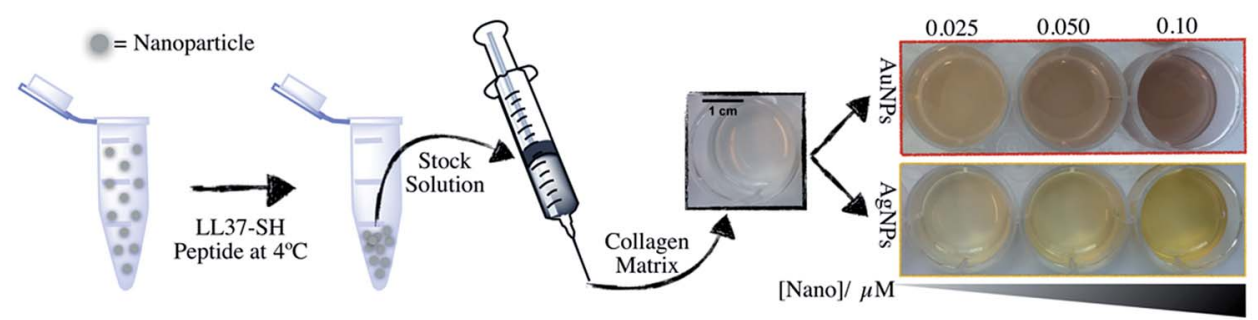

b

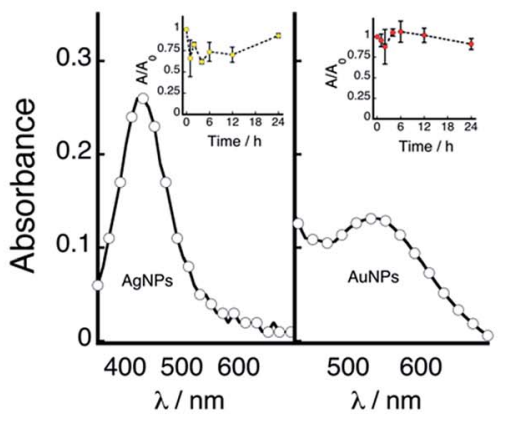

C
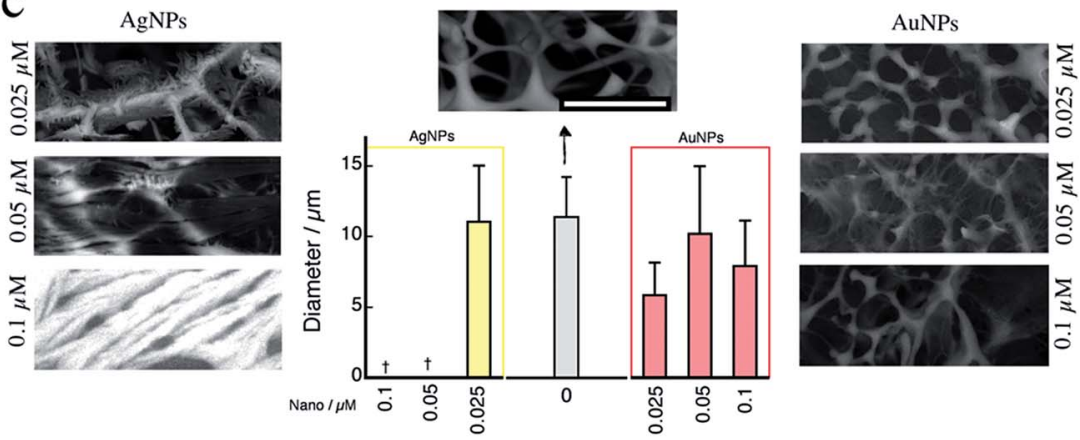

d

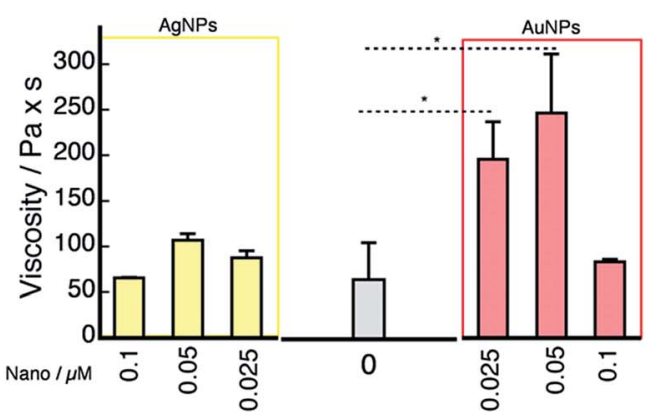

e

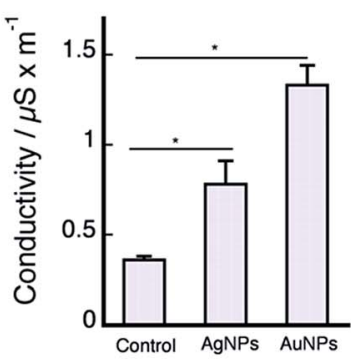

Fig. 1 (a) Schematic representation of the protocol used for nanoparticle incorporation within the collagen matrices (see ESI for further details $\uparrow$ ). Images on the right show the colour of the gels containing the nanoparticles at three different concentrations $(0.025,0.05$, and $0.1 \mu \mathrm{M})$. (b) Left: Representative absorption spectra for collagen hydrogels containing $0.1 \mu \mathrm{M}$ of either nanosilver (left) or nanogold (right). Insets: changes in the plasmonic absorbance of the samples measured at different time points for samples incubated at $37^{\circ} \mathrm{C}$ in $\mathrm{PBS}$ buffer. (c) Representative $\mathrm{CRYO}$ SEM images for collagen matrices prepared with different nanosilver concentrations (left panel), or with different concentrations of nanogold (right panel). Middle: Average pore size for the different collagen hydrogels. Note that for the samples prepared using 0.05 and $0.1 \mu \mathrm{M}$ of nanosilver, no porous structure was observed; rather, a fibrous-like structure formed. Representative CRYO-SEM image for the control hydrogel has been included in the middle. † Denotes that pore size measurement was not performed due to lack of identifiable pores. (d) Hydrogel viscosity for samples prepared without (grey bar), and with different concentrations of nanosilver measured at $25^{\circ} \mathrm{C}$ (performed in quadruplicate). (e) Electrical conductivity of collagen hydrogels without and with $0.025 \mu \mathrm{M}$ of nanosilver or nanogold. * $p<0.05$ calculated from two-tailed $t$-test $(n=4)$.

nanoparticles affected the average pore size of the matrices when compared to the control without nanoparticles (Fig. 1c, middle). Similarly, no changes in the denaturation temperature, water content, or amide IR vibration were observed for any of the samples (Fig. S3 $\dagger$ ). These findings indicate that the nanoparticulated composites did not affect the degree of crosslinking and/or hydrophobicity of the material. Interestingly, collagenase degradation rate for the collagen matrices containing nanogold were considerably slower when compared to either the control and/or those containing nanosilver (Fig. S4†). This could be attributed to enzyme deactivation in the presence of stable nanogold released upon collagen matrix degradation. ${ }^{16}$

Changes in the materials' viscosity were also evaluated by rheometry (Fig. 1d). The addition of nanosilver does not alter the materials viscosity at any of the tested concentrations.
However, the presence of nanogold led to an increase in viscosity of $\approx 4 \times$ at $0.025 \mu \mathrm{M}$ and $\approx 5 \times$ at $0.05 \mu \mathrm{M} v$ s. the control. This increase in viscosity was not observed at $0.1 \mu \mathrm{M}$ nanogold, a factor which may be linked to modifications in the micro-structure (i.e. collagen assembly) of the material. Finally, composite macroscopic conductivity was measured for samples containing $0.025 \mu \mathrm{M}$ of either silver or gold nanoparticles (Fig. 1e). Interestingly, despite the low concentration of nanoparticles, conductivity was increased by 2- and 3-fold for AgNPs and AuNPs, respectively. This increase resembles what we have recently reported for the preparation of other collagen-nanosilver composites; ${ }^{3}$ however, in the present study, measurements were carried out on hydrated gels, which more closely mimics physiological conditions. 
The materials containing nanosilver, which has been reported to exert toxic effects in cells, ${ }^{19}$ were screened for their toxic effect using cardiac fibroblasts (Fig. S5†). The only material that did not hinder cell proliferation was the one containing a concentration of $0.025 \mu \mathrm{M}$ nanosilver. Therefore, only this concentration of nanosilver was used in all the subsequent experiments of cell compatibility and used for comparison with the nanogold samples. No toxicity was observed in cultured cardiomyocytes cells upon incubation with the nanogold, see Fig. S6. $\dagger$

A significant body of literature has reported on the effects nanoparticulated materials have on immune/inflammatory cells. However, the majority of these studies have been carried out in solution. ${ }^{20-24}$ A more biologically relevant model for biomimetic matrices intended for supporting and promoting cell infiltration would include evaluation of the migration and polarization of macrophages, which are critical in the body's immune/inflammatory response to implanted biomaterials. Such responses will ultimately dictate degradation and bioincorporation of the matrix within the host. Note that our composite materials containing nanosilver did not promote native macrophage polarization into a M1 (pro-inflammatory) or M2 (pro-healing) phenotype (Fig. S7†). However, migration assays for non-polarized and polarized macrophages showed that the matrices containing silver or gold nanoparticles at the lowest concentration tested promoted greater M2 infiltration by 1.5- and 2.2-fold, respectively (Fig. 2). The underlying mechanisms involved in this enhancement may be related to microscopic modifications of the matrix surface. As graphically depicted in the CRYO-SEM images shown in Fig. 1c, pore sizes
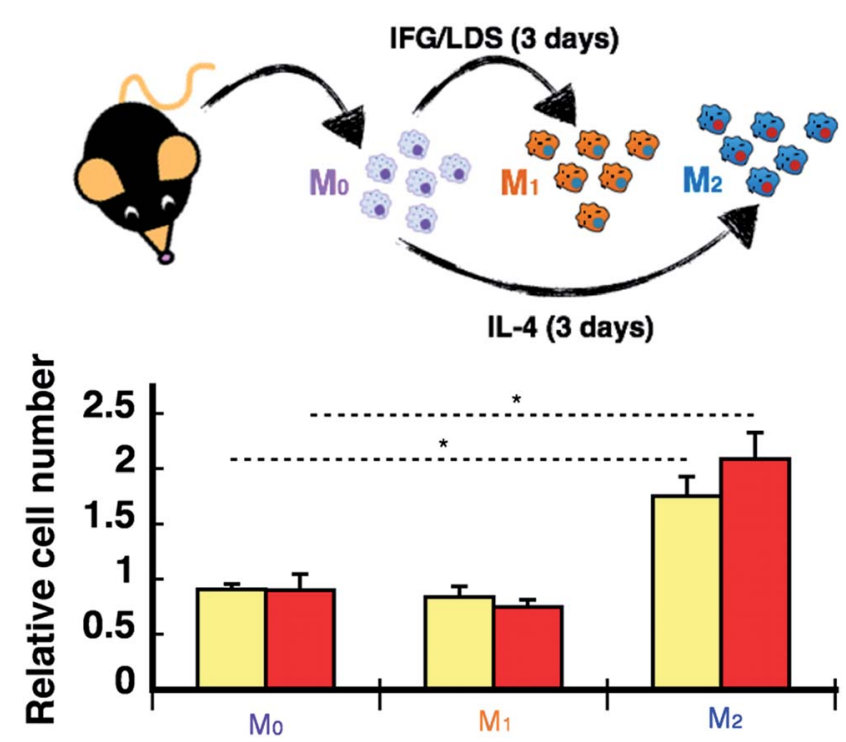

Fig. 2 Top: Schematic representation of the macrophage protocol used for testing the immune/inflammatory effects of the hybrid matrices. Bottom: Changes in relative cell number compared to control group for different polarization states of murine macrophages cultured on silver (yellow bars) and gold (red bars) containing matrices. Data were collected in quadruplicate. Error bars correspond to standard errors calculated from counting 12 different migratory areas, see Experimental. *represents $p<0.05$ from $t$-tests. were not affected by the presence of the nanomaterials, however some distinctive microscopic features were seen, particularly for the sample containing nanosilver. It is these differences which may promote preferential mobility of M2 macrophages within the collagen matrix.

Fig. 3 shows representative immuno-fluorescence staining for $\alpha$-sarcomeric actin ( $\alpha$-SA) and connexin-43 in neonatal cardiomyocytes cultured on the different collagen matrix formulations tested in this study. In all cases, cells displayed the characteristic expression pattern of $\alpha$-SA. Furthermore, adding nanoparticles to the collagen matrix did not affect the number of cells that expressed connexin-43 under normal cell culture

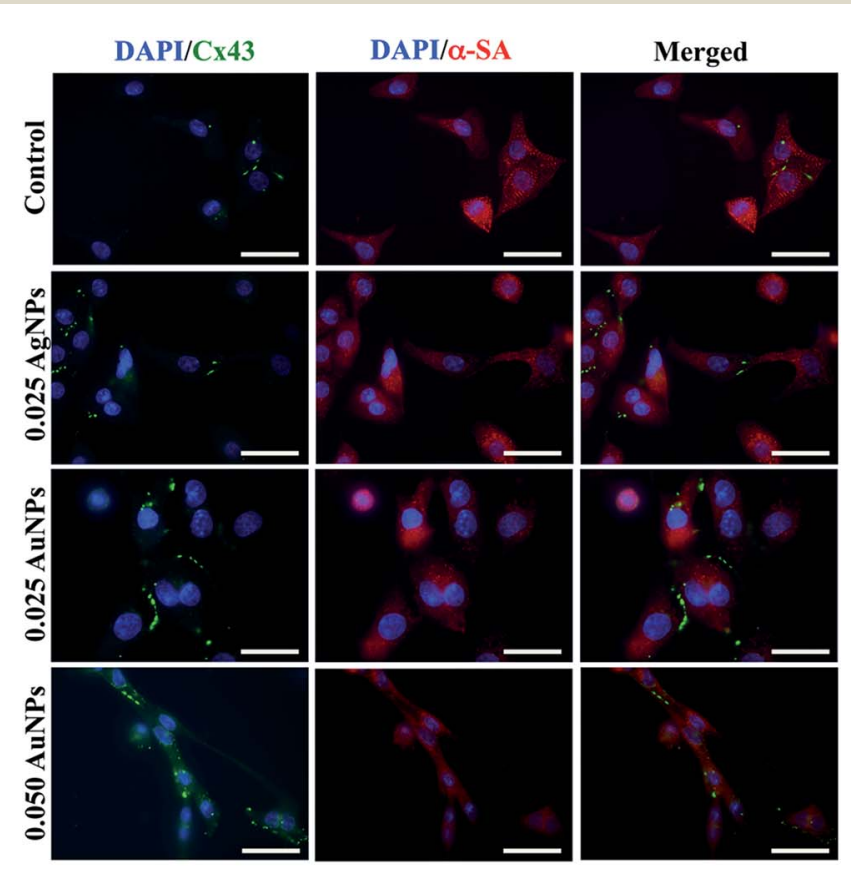

(+) CPACE

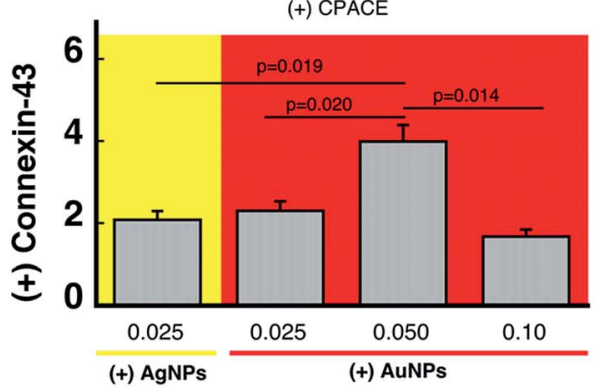

Fig. 3 Top: Representative confocal immunofluorescence microscopy images of neonatal cardiomyocytes seeded onto the matrices without and with the nanomaterials at different concentrations in $\mu \mathrm{M}$. Cells were subjected to electrical stimulation in CPACE for $24 \mathrm{~h}(0.4 \mathrm{~V}$, $5 \mathrm{~ms}$ pulse duration and $5 \mathrm{~Hz}$ frequency). After fixation, cells were stained for nuclei (DAPI), alpha-sarcomeric actinin ( $\alpha$-SA) and connexin-43 (Cx43). Images of the merged channels are also provided. Scale bar $=50 \mu \mathrm{m}$. Bottom: Relative number to control without nanoparticles of $\mathrm{Cx} 43^{+}$foci per cell for cells exposed to CPACE. In all cases $>50$ cells were counted from different areas and from at least three slides, from different experiments of the same group. Error bars correspond to standard errors. $P$-values were calculated from $t$-tests for 2 groups with unequal variance. Control groups were cells seeded onto collagen hydrogels containing no nanoparticles. 
conditions (Fig. S8 $\dagger$ ). However, upon electrical stimulation at $5 \mathrm{~Hz}$ and $0.4 \mathrm{~V}$ for $24 \mathrm{~h}$, there was an increase of $2 \times$ in the connexin-43 levels for the samples containing $0.025 \mu \mathrm{M}$ silver or gold when compared to the control samples without nanoparticles ( $p<0.01$, Fig. 3 bottom). While increasing the concentration of gold to $0.05 \mu \mathrm{M}$ produced an increase in the connexin levels of up to $4 \times(p<0.05) v s$. control group (Fig. 3 bottom), further increases in gold concentration produced a decline in connexin-43 to levels similar to the lowest gold concentration, which resembles the trend observed for the viscosity. This again can be linked to loss in the supramolecular arrangement of the collagen fibres at high nanogold concentrations. Note that connexin-43 levels were also recently reported by our team to be increased in cardiomyocytes cultured on collagen fibres prepared with collagen-coated nanosilver as an electroconductive doping agent. ${ }^{3}$ In the present work, we achieved a similar effect by using a soft collagen matrix containing nanogold prepared in a one-pot reaction system.

\section{Conclusions}

While the use of nanomaterials for biomedical applications has exponentially increased over the last decade, the development of thermo-crosslinkable electroconductive materials for cardiac tissue repair has been less widely explored. Our findings are interesting from a practical viewpoint as they identify that the addition of micromolar concentrations of metal nanoparticles can alter the supramolecular structure of the biomimetic collagen matrix. However, such changes were only structural and did not affect the macroscopic properties of the materials. The incorporation of nanogold led to an increase in the mechanical properties of the composite materials ( $\approx 5$ times). Furthermore, the composite materials containing the nanoparticles favoured the migration of pro-healing M2 macrophages compared to the other macrophage phenotypes, which may contribute to the prohealing properties reported in the literature for gold and silver nanoparticles. ${ }^{17,25-27}$ In addition, despite the fact that silver is a much better electroconductive element than gold, the assembly of gold nanoparticles within the collagen matrix produced the material with superior conductivity and capabilities for promoting connectivity in cardiomyocytes.

\section{Conflicts of interest}

No conflicts of interest to be declared.

\section{Acknowledgements}

This work was made possible by funding from the Natural Sciences and Engineering Research Council (NSERC) to EIA and EJS, RGPIN-2015-0632. EIA also thanks UOHI start-up grant 1255. EIA, EJS and WL thank the Canadian Institutes of Health Research for Project Grants. FV acknowledges support from NSERC, the Canada Foundation for Innovation and the Ontario Ministry of Research and Innovation through the Leaders of Opportunity fund. KH acknowledges the Tohoku Development Memorial Foundation and the Student Exchange Support
Program from Japan Student Services Organization. The authors would like to thank Professor May Griffith from University of Montreal for providing a sample of the LL37 peptide. Cell isolations were conducted with ethical approval from the University of Ottawa Animal Care Committee and in compliance with the National Institutes of Health Guide for the Use of Laboratory Animals. The authors would to also thank Dr Brian McNeill for his technical assistance in the macrophage experiments.

\section{References}

1 R. Xing, K. Liu, T. Jiao, N. Zhang, K. Ma, R. Zhang, Q. Zou, G. Ma and X. Yan, Adv. Mater., 2016, 28, 3669-3676.

2 R. Xing, T. Jiao, L. Yan, G. Ma, L. Liu, L. Dai, J. Li, H. Möhwald and X. Yan, ACS Appl. Mater. Interfaces, 2015, 7, 24733-24740.

3 S. Allison, M. Ahumada, C. Andronic, B. McNeill, F. Variola, M. Griffith, M. Ruel, V. Hamel, W. Liang, E. J. Suuronen and E. I. Alarcon, J. Mater. Chem. B, 2017, 5, 2402-2406.

4 S. R. Shin, S. M. Jung, M. Zalabany, K. Kim, P. Zorlutuna, S. b. Kim, M. Nikkhah, M. Khabiry, M. Azize, J. Kong, K.-t. Wan, T. Palacios, M. R. Dokmeci, H. Bae, X. Tang and A. Khademhosseini, ACS Nano, 2013, 7, 2369-2380.

5 V. Martinelli, G. Cellot, F. M. Toma, C. S. Long, J. H. Caldwell, L. Zentilin, M. Giacca, A. Turco, M. Prato, L. Ballerini and L. Mestroni, Nano Lett., 2012, 12, 1831-1838. 6 J. Zhou, J. Chen, H. Sun, X. Qiu, Y. Mou, Z. Liu, Y. Zhao, X. Li, Y. Han, C. Duan, R. Tang, C. Wang, W. Zhong, J. Liu, Y. Luo, M. Xing and C. Wang, Sci. Rep., 2014, 4, 3733.

7 T. Dvir, B. P. Timko, M. D. Brigham, S. R. Naik, S. S. Karajanagi, O. Levy, H. Jin, K. K. Parker, R. Langer and D. S. Kohane, Nat. Nanotechnol., 2011, 6, 720-725.

8 M. Shevach, S. Fleischer, A. Shapira and T. Dvir, Nano Lett., 2014, 14, 5792-5796.

9 A. Orza, O. Soritau, L. Olenic, M. Diudea, A. Florea, D. Rus Ciuca, C. Mihu, D. Casciano and A. S. Biris, ACS Nano, 2011, 5, 4490-4503.

10 P. Baei, S. Jalili-Firoozinezhad, S. Rajabi-Zeleti, M. TafazzoliShadpour, H. Baharvand and N. Aghdami, Mater. Sci. Eng., C, 2016, 63, 131-141.

11 M. M. Nguyen, N. C. Gianneschi and K. L. Christman, Curr. Opin. Biotechnol., 2015, 34, 225-231.

12 E. I. Alarcon, B. Vulesevic, A. Argawal, A. Ross, P. Bejjani, J. Podrebarac, R. Ravichandran, J. Phopase, E. J. Suuronen and M. Griffith, Nanoscale, 2016, 8, 6484-6489.

13 E. I. Alarcon, K. Udekwu, M. Skog, N. L. Pacioni, K. G. Stamplecoskie, M. Gonzalez-Bejar, N. Polisetti, A. Wickham, A. Richter-Dahlfors, M. Griffith and J. C. Scaiano, Biomaterials, 2012, 33, 4947-4956.

14 S. Jockusch, M. S. Landis, B. Freiermuth and N. J. Turro, Macromolecules, 2001, 34, 1619-1626.

15 K. G. Stamplecoskie and J. C. Scaiano, J. Am. Chem. Soc., 2010, 132, 1825-1827.

16 K. L. McGilvray, M. R. Decan, D. Wang and J. C. Scaiano, J. Am. Chem. Soc., 2006, 128, 15980-15981. 
17 S. McLaughlin, M. Ahumada, W. Franco, T.-F. Mah, R. Seymour, E. J. Suuronen and E. I. Alarcon, Nanoscale, 2016, 8, 19200-19203.

18 M. Vignoni, H. de Alwis Weerasekera, M. J. Simpson, J. Phopase, T. F. Mah, M. Griffith, E. I. Alarcon and J. C. Scaiano, Nanoscale, 2014, 6, 5725-5728.

19 H. de Alwis Weerasekera, M. Griffith and E. I. Alarcon, in Silver Nanoparticle Applications: In the Fabrication and Design of Medical and Biosensing Devices, ed. E. I. Alarcon, K. Udekwu and M. Griffith, Springer, UK, 2015, pp. 93-125. 20 A. Paul, H. Ju, S. Rangasamy, Y. Shim and J. M. Song, Nanoscale Res. Lett., 2015, 10, 140.

21 Z. Shavandi, T. Ghazanfari and K. N. Moghaddam, Immunopharmacol. Immunotoxicol., 2011, 33, 135-140.

22 J. Park, D. H. Lim, H. J. Lim, T. Kwon, J. S. Choi, S. Jeong, I. H. Choi and J. Cheon, Chem. Commun., 2011, 47, 43824384.
23 J. Malaczewska, R. Wojcik, E. Kaczorek, W. Rekawek and A. K. Siwicki, Res. Vet. Sci., 2017, 110, 4-11.

24 K. Niikura, T. Matsunaga, T. Suzuki, S. Kobayashi, H. Yamaguchi, Y. Orba, A. Kawaguchi, H. Hasegawa, K. Kajino, T. Ninomiya, K. Ijiro and H. Sawa, ACS Nano, 2013, 7, 3926-3938.

25 S. Pothireddy, A. Kaliki, A. R. Mekapogu, M. Yegireddy, E. P. Pagadala and T. N. Prasad, IET Nanobiotechnol., 2016, 10, 340-348.

26 X. Yang, J. Yang, L. Wang, B. Ran, Y. Jia, L. Zhang, G. Yang, H. Shao and X. Jiang, ACS Nano, 2017, 11, 5737-5745.

27 X. Li, H. Wang, H. Rong, W. Li, Y. Luo, K. Tian, D. Quan, Y. Wang and L. Jiang, J. Colloid Interface Sci., 2015, 445, 312-319. 